\title{
COMPARAÇÃO ENTRE MODELOS INTEGRADOS DE USO DO SOLO E TRANSPORTES APLICANDO FERRAMENTAS DE ANÁLISE DE CONTEÚDO
}

Comparison between land use and transport integrated models applying content analysis tools

Comparación de modelos integrados de uso de la tierra y transporte con aplicación de las herramientas de análisis de contenido

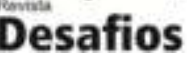

Artigo Original

Original Article

Artículo Original

\section{Lílian dos Santos Fontes Pereira Bracarense ${ }^{* 1}$, Pastor Willy Gonzales Taco ${ }^{2}$}

${ }^{1}$ Curso de Engenharia Civil, Universidade Federal do Tocantins, Palmas, Brasil

${ }^{2}$ Programa de Pós-Graduação em Transportes, Universidade de Brasília, Brasília, Brasil

*Correspondência: Curso de Engenharia Civil, Av. NS 15, 109 Norte, Palmas, Tocantins, Brasil. CEP:77.010-090. e-mail lilianfontes@uft.edu.br

Artigo recebido em 25/01/2017 aprovado em 04/04/2017 publicado em 19/04/2017.

\section{RESUMO}

A escolha de um modelo a ser adotado no processo de planejamento urbano e de transportes requer amplas análises acerca das características do modelo e adequação ao problema a ser tratado. Esse artigo apresenta a comparação entre vinte e dois modelos integrados de uso do solo e transportes por meio da utilização de métodos de análise de conteúdo para simplificar esse processo e auxiliar o planejador a definir o modelo a ser adotado. Os resultados mostraram que os métodos avaliados possuem potencial de elencar as principais informações e estabelecer conexões importantes, simplificando a análise de grandes volumes de informação. Além disso, a comparação permitiu identificar classes de semelhanças entre os modelos e diagnosticar limitações, vislumbrando novas oportunidades de pesquisa na área, como aplicações em estudos regionais.

Palavras-chave: modelos de uso do solo e transportes; análise de conteúdo.

\section{ABSTRACT}

The choice of a model to be adopted in the process of urban planning and transportation requires extensive analyzes of model characteristics and suitability to the problem being treated. This paper presents a comparison of twenty-two integrated models of land use and transportation by using content analysis methods to simplify this process and assist the planner to define the model to be adopted. The results showed that the evaluated methods have the potential to list the key information and establish important connections, simplifying the analysis of large volumes of information. In addition, the comparison has identified classes of similarities between the models and diagnose limitations, seeing new research opportunities in the area, such as applications in regional studies.

Keywords: land use and transportation models; content analysis.

\section{RESUMEN}

La elección de un modelo que ha de adoptarse en el proceso de planificación urbana y de transporte requiere extensos análisis de las características del modelo y la idoneidad para el problema que se está tratando. En este trabajo se presenta una comparación de veinte y dos modelos integrados de uso de la tierra y el transporte mediante el uso de métodos de análisis de contenido para simplificar este proceso y ayudar al planificador para definir el modelo a adoptar. Los resultados mostraron que los métodos evaluados tienen el potencial de una lista de la información clave y establecer conexiones importantes, lo que simplifica el análisis de grandes volúmenes de información. Además, la comparación ha identificado clases de similitudes entre los modelos y diagnosticar las 
limitaciones, viendo nuevas oportunidades de investigación en el área, tales como aplicaciones en estudios regionales.

Descriptores: modelos integrados de uso de la tierra y transporte; análisis de contenido.

\section{INTRODUÇÃO}

A análise da interação entre transportes e uso do solo é um aspecto delicado, e se mostra como um aspecto chave na definição de políticas públicas. Existe um potencial de complementaridade entre as políticas de uso do solo e transportes como forma de atenuar problemas de saturação das infraestruturas urbanas e de transportes. Como suporte para essas análises, os modelos integrados de uso do solo e transportes têm sido aplicados em diversas cidades no mundo.

Os modelos integrados buscam incorporar a percepção de que o uso do solo também é afetado pela oferta de transportes, assim como o uso do solo afeta os transportes. Esta realimentação indica que ambos estão mutuamente interligados, diferentemente dos modelos convencionais, que têm apenas o efeito direto.

Diversos modelos e estudos têm sido desenvolvidos na academia para a modelagem integrada de uso do solo e transporte urbano, e têm sido empregados no planejamento de várias cidades no mundo, como a aplicação do TRANUS nas cidades de Swindon / Inglaterra (MODELÍSTICA, 2009), Aveiro / Portugal (BANDEIRA, 2009), Sapporo / Japão (VICHIENSAN et al., 2003) e Oregon / EUA (PARSONS E URBAN ANALYTICS, 1999), Belo Horizonte / Brasil (PEREIRA e OLIVEIRA, 2014).

Embora partam do mesmo princípio, existem diferenças significativas entre os modelos quanto à finalidade, aos dados requeridos, à escala de aplicação, à dificuldade de calibração entre outros fatores. A obtenção de informação completa acerca dos requisitos de um determinado modelo pode demandar tempo e aprofundamento nos estudos, ainda que o mesmo venha a ser descartado. Em função dessa variedade, a decisão de qual modelo empregar em um estudo pode não ser tão simples, e ao mesmo tempo, a escolha errada pode comprometer o resultado, por não atender adequadamente às necessidades específicas do estudo. Além disso, apesar de uma grande variedade de estudos e modelos em desenvolvimento, algumas limitações como a disponibilidade de dados desagregados e complexidade da construção dos modelos ainda dificultam a aplicação das análises integradas de uso do solo e transportes de forma mais abrangente nos estudos de transportes.

Nesse contexto, esse trabalho tem o objetivo de discutir as semelhanças, potencialidades e limitações de vinte e dois modelos integrados de uso do solo e transportes a partir da aplicação de uma ferramenta de análise de conteúdo. Inicialmente é apresentada uma revisão bibliográfica abordando dados gerais sobre os modelos a serem estudados. Em seguida apresenta-se a metodologia de análise e os requisitos de aplicação. Por fim, são apresentados e discutidos os resultados obtidos, identificando-se novas possibilidades de aplicação dos modelos para estudos futuros.

\section{MODELOS INTEGRADOS DE USO DO SOLO E TRANSPORTE}

Os modelos integrados exploram a interação entre transportes, localização de atividades e uso do solo. Em modelos tradicionais, o uso do solo é definido exogenamente, e a partir dessa localização das atividades definem-se os fluxos, deslocamentos e a demanda por transportes. No modelo integrado, o 
uso do solo também é modelado endogenamente, sendo afetado pela política de transportes, e também a afetando, numa interação em ciclos até atingir-se um equilíbrio (PEREIRA E OLIVEIRA, 2014).

Além da vantagem de medir a influência dos transportes no uso do solo, a incorporação do uso do solo permite a simulação dos impactos de mudanças na regulação urbana, tais como flexibilização dos limites de área construída.

Os modelos de atividades reconhecem a existência de complexas interações entre a participação em atividades e o padrão da viagem (MODELÍSTICA, 2009). Este tipo de modelo, com ênfase na participação em atividades e nos seus padrões de realização, pode fornecer informações sobre o modo como os indivíduos e os domicílios alteram esta participação (atividades realizadas, modos de viagem, etc.) em resposta a determinada política de transporte e/ou de ocupação do solo, o que permite prever com mais precisão a dinâmica urbana da cidade.

Feil et. al (2009) relaciona as três principais linhas de pesquisa em modelagem de demanda baseada em atividades:
Modelos econométricos: utilizam sistemas de equações para buscar relações explicativas entre atributos.

Utility-based microsimulations: modelos que aplicam processo sequencial de tomada de decisão.

Computational process models (CPMs): substituem a maximização da utilidade por processamento de informação e tomada de decisão. Os principais modelos nessa linha abordam princípios comportamentais de aquisição e representação.

Para uma visão geral, vinte e dois modelos de uso do solo e de transportes urbanos foram selecionados da publicação US EPA (2000) para uma análise comparativa (Tabela 1). São apresentados modelos desenvolvidos por pesquisadores de universidades americanas e europeias, além de empresas de consultoria. Vários dos modelos descritos não são comerciais, mas objeto de pesquisas com aplicações em casos específicos. Os modelos distinguem-se em relação ao objeto, às variáveis utilizadas e resultados possíveis. A Tabela 1 apresenta ainda uma breve análise crítica considerando potencialidades e limitações de cada um.

Tabela 1. Modelos avaliados.

\begin{tabular}{|c|c|c|}
\hline Modelo & Descrição & Características - Vantagens e limitações \\
\hline $\begin{array}{l}\text { California Urban } \\
\text { Futures (CUF) } \\
\text { Model: CUF-1 }\end{array}$ & $\begin{array}{l}\text { Desenvolvido por John Landis, } \\
\text { Institute of Urban and Regional } \\
\text { Planning, University of California at } \\
\text { Berkeley. Permite simular como o } \\
\text { crescimento e desenvolvimento de } \\
\text { políticas pode alterar a localização, } \\
\text { padrão e intensidade de } \\
\text { desenvolvimento urbano. }\end{array}$ & $\begin{array}{l}\text { O modelo permite simular o crescimento populacional } \\
\text { baseando-se em cenários de políticas públicas. Porém, } \\
\text { o crescimento urbano é considerado } \\
\text { predominantemente periférico, o que inviabiliza } \\
\text { simulações de adensamento. Também não permite } \\
\text { analisar a influência de alterações na infraestrutura de } \\
\text { transportes nos padrões de uso do solo, apenas algumas } \\
\text { interferências que mudanças no uso do solo podem } \\
\text { gerar na demanda de transportes. Além disso, o modelo } \\
\text { é restrito a simular o desenvolvimento do uso do solo } \\
\text { residencial. }\end{array}$ \\
\hline $\begin{array}{l}\text { California Urban } \\
\text { Futures (CUF) } \\
\text { Model: CUF-2 }\end{array}$ & $\begin{array}{l}\text { Dos mesmos desenvolvedores do CUF- } \\
\text { 1, apresenta alguns avanços teóricos. }\end{array}$ & $\begin{array}{l}\text { Traz inovações ao modelo CUF-1, como a } \\
\text { possibilidade de projetar o número de empregos, e } \\
\text { amplia a possibilidade de simulação de diferentes usos } \\
\text { do solo, além de permitir a calibração com dados }\end{array}$ \\
\hline
\end{tabular}




\begin{tabular}{|c|c|c|}
\hline Modelo & Descrição & Características - Vantagens e limitações \\
\hline & & $\begin{array}{l}\text { históricos. Também traz melhorias em relação as } \\
\text { análises de transporte e uso do solo, porém requer uma } \\
\text { quantidade muito maior de dados e profundo } \\
\text { conhecimento estatístico para efetuar a calibração. }\end{array}$ \\
\hline $\begin{array}{l}\text { California Urban } \\
\text { and Biodiversity } \\
\text { Analysis Model } \\
\text { (CURBA) }\end{array}$ & $\begin{array}{l}\text { Dos mesmos desenvolvedores de CUF- } \\
1 \text { e CUF-2, avalia os possíveis efeitos } \\
\text { de alternativas de padrões e políticas de } \\
\text { crescimento urbano na biodiversidade } \\
\text { e qualidade de ambiente natural. }\end{array}$ & $\begin{array}{l}\text { O modelo não diferencia os tipos de uso do solo } \\
\text { urbano, e as análises relacionadas à transportes } \\
\text { limitam-se à interferência da infraestrutura de } \\
\text { transporte no uso do solo. }\end{array}$ \\
\hline DELTA & $\begin{array}{l}\text { Pacote de modelagem econômica / uso } \\
\text { do solo desenvolvido por Davids } \\
\text { Simmonds Consultancy, Cambridge, } \\
\text { UK. Representa o mercado de solo com } \\
\text { preços endógenos em cada período. }\end{array}$ & $\begin{array}{l}\text { Pode ser implementado junto com um modelo de } \\
\text { transportes e fornecer impactos da mudança do uso do } \\
\text { solo na demanda e impactos do aumento de } \\
\text { acessibilidade na localização de atividades. Permite a } \\
\text { modelagem de diferentes usos urbanos. }\end{array}$ \\
\hline $\begin{array}{l}\text { Disaggregated } \\
\text { Residential } \\
\text { Allocation Model } \\
\text { of household } \\
\text { Location and the } \\
\text { Employment } \\
\text { Allocation Model } \\
\text { (DRAM/EMPAL) }\end{array}$ & $\begin{array}{l}\text { Elaborado por S.H. Putman and } \\
\text { Associates, Inc. Projeta as interações e } \\
\text { distribuição de empregos e habitação } \\
\text { em uma área geográfica específica. }\end{array}$ & $\begin{array}{l}\text { É um modelo que requer dados geralmente disponíveis, } \\
\text { e trabalha de forma mais agregada, com um processo } \\
\text { de calibração relativamente simples. Não permite } \\
\text { simular outras políticas de desenvolvimento além de } \\
\text { zoneamento, e as simplificações nos dados requeridos } \\
\text { refletem resultados menos sensíveis a escolhas } \\
\text { individuais. }\end{array}$ \\
\hline $\begin{array}{l}\text { Growth Simulation } \\
\text { Model (GSM) }\end{array}$ & $\begin{array}{l}\text { Projeta crescimento da população e } \\
\text { efeitos de desenvolvimento no uso e } \\
\text { ocupação do solo. }\end{array}$ & $\begin{array}{l}\text { Permite uma análise das mudanças no uso do solo } \\
\text { considerando preferências de mercado e é possível de } \\
\text { ser aplicado em diferentes escalas. As adaptações } \\
\text { necessárias para novas aplicações demandam } \\
\text { habilidades de programação e não fornece mapas do } \\
\text { uso do solo em formato vetorial. }\end{array}$ \\
\hline INDEX & $\begin{array}{l}\text { Mede as características e performance } \\
\text { dos planos de uso do solo e desenho } \\
\text { urbano com indicadores a partir das } \\
\text { políticas e objetivos comunitários. }\end{array}$ & $\begin{array}{l}\text { Não modela o impacto da infraestrutura de transportes } \\
\text { no uso do solo, nem as interferências do uso do solo na } \\
\text { demanda de transportes, portanto depende de estar } \\
\text { conectado a um modelo de transportes para estimar } \\
\text { esses impactos. Requer uma base de dados detalhada. }\end{array}$ \\
\hline IRPUD & $\begin{array}{l}\text { Modelo da região de Dortmund } \\
\text { desenvolvido na Universidade de } \\
\text { Dortmund. Projeta o impacto de } \\
\text { mudanças econômicas e tecnológicas } \\
\text { de longo prazo na habitação, } \\
\text { transporte, políticas públicas, uso do } \\
\text { solo e infraestrutura. }\end{array}$ & $\begin{array}{l}\text { O modelo é considerado dinâmico e permite a } \\
\text { completa análise integrada dos efeitos mútuos entre uso } \\
\text { do solo e transportes. Requer uma grande variedade de } \\
\text { parâmetros e não inclui a simulação de transporte } \\
\text { urbano de cargas. }\end{array}$ \\
\hline $\begin{array}{l}\text { Land } \\
\text { Transformation } \\
\text { Model (LTM) }\end{array}$ & $\begin{array}{l}\text { Desenvolvido por Dr. Bryan C. } \\
\text { Pijanowski,da Michigan State } \\
\text { University. Utiliza padrões de interação } \\
\text { para prever mudanças no uso do solo. }\end{array}$ & $\begin{array}{l}\text { O modelo abrange diferentes usos do solo não urbanos, } \\
\text { mas os usos urbanos se limitam ao residencial. Não } \\
\text { permite simular os efeitos da mudança de uso do solo } \\
\text { na demanda de transportes. É de difícil implementação. }\end{array}$ \\
\hline $\begin{array}{l}\text { Land-Use Change } \\
\text { Analysis System } \\
\text { (LUCAS) }\end{array}$ & $\begin{array}{l}\text { Examina o impacto de atividades } \\
\text { humanas no uso do solo e consequentes } \\
\text { impactos no meio ambiente e recursos } \\
\text { naturais. }\end{array}$ & $\begin{array}{l}\text { O modelo permite simular o efeito da infraestrutura de } \\
\text { transportes sobre o uso do solo, mas não o contrário. É } \\
\text { de difícil calibração. }\end{array}$ \\
\hline $\begin{array}{l}\text { Markov Model of } \\
\text { Residential } \\
\text { Vacancy Transfer }\end{array}$ & $\begin{array}{l}\text { Elaborado por Philip Emmi and Lena } \\
\text { Magnusson. Explora mudanças na } \\
\text { demanda de vários tipos de habitação }\end{array}$ & $\begin{array}{l}\text { É um modelo matemático aplicável a cidades de } \\
\text { pequeno porte. Não permite a simulação da interface } \\
\text { uso do solo e transportes e é limitado à modelagem do }\end{array}$ \\
\hline
\end{tabular}




\begin{tabular}{|c|c|c|}
\hline Modelo & Descrição & Características - Vantagens e limitações \\
\hline & residencial dentro de uma comunidade. & $\begin{array}{l}\text { uso residencial. Permite avaliar o impacto de vazios } \\
\text { urbanos e acomodação de novos habitantes de acordo } \\
\text { com o mercado imobiliário. Requer série de dados } \\
\text { históricos. }\end{array}$ \\
\hline MEPLAN & $\begin{array}{l}\text { Pacote de modelo integrado de uso do } \\
\text { solo e transportes desenhado para } \\
\text { comparar propostas de planos e } \\
\text { políticas públicas. Representa o } \\
\text { mercado de solo com preços endógenos } \\
\text { em cada período. }\end{array}$ & $\begin{array}{l}\text { Abrange três módulos: econômico/uso do solo; } \\
\text { transportes; e avaliação econômica. Permite uma } \\
\text { flexibilidade quanto aos dados de entrada requeridos. } \\
\text { Permite análise de diferentes políticas públicas. O } \\
\text { processo de calibração é complicado e demorado e a } \\
\text { escassez de dados pode dificultar a validação de dados } \\
\text { do ano base. }\end{array}$ \\
\hline METROSIM & $\begin{array}{l}\text { Modelo microeconômico de uso do } \\
\text { solo e transportes desenvolvido para a } \\
\text { região metropolitana de Nova Iorque. } \\
\text { Representa o mercado de solo com } \\
\text { preços endógenos em cada período. } \\
\text { Usa uma abordagem econômica de } \\
\text { previsão interdependente de efeitos de } \\
\text { transporte e sistemas de uso do solo. }\end{array}$ & $\begin{array}{l}\text { Bem completo em termos de possibilidade de } \\
\text { simulação de usos do solo e impactos na interface com } \\
\text { sistemas de transporte. Flexível em relação ao formato } \\
\text { dos dados de saída. Tem um perfil de análise } \\
\text { econômica voltado para influência das forças de } \\
\text { mercado no uso do solo. }\end{array}$ \\
\hline $\begin{array}{l}\text { Sub-Area } \\
\text { Allocation Model- } \\
\text { Improved Method } \\
\text { (SAM-IM) } \\
\text { (formally LAM) }\end{array}$ & $\begin{array}{l}\text { Desenvolvido pela Maricopa (Arizona) } \\
\text { Association os Governments. Cria } \\
\text { novos cenários de uso do solo que } \\
\text { refletem conceitos de desenvolvimento } \\
\text { alternativos para o futuro. }\end{array}$ & $\begin{array}{l}\text { O processo de urbanização é simulado pela avaliação } \\
\text { de solo disponível para absorver o crescimento, } \\
\text { considerando disponibilidade de infraestrutura, } \\
\text { consistência com o zoneamento e políticas de } \\
\text { desenvolvimento locais. Bem integrado com sistemas } \\
\text { de informação geográfica, permite avaliar uma } \\
\text { diversidade de usos urbanos e não urbanos e sua } \\
\text { influência recíproca nos sistemas de transportes. } \\
\text { Requer um conhecimento altamente especializado para } \\
\text { operacionalizar o modelo. }\end{array}$ \\
\hline $\begin{array}{l}\text { The SLEUTH } \\
\text { Model (formally } \\
\text { Clarke Cellular } \\
\text { Automata) }\end{array}$ & $\begin{array}{l}\text { Projeta crescimento urbano e examina } \\
\text { como novas áreas consomem o entorno } \\
\text { e impactam o meio ambiente. } \\
\text { (KRAMER, 1996) }\end{array}$ & $\begin{array}{l}\text { Permite avaliar uma grande variedade de usos e como } \\
\text { são impactados pela oferta de transportes. Permite } \\
\text { diferenciar quatro tipos de crescimento: espontâneo, } \\
\text { difuso, orgânico ou influenciado por eixos viários. Não } \\
\text { aborda demanda de transportes, população, ou } \\
\text { impactos econômicos e políticos. }\end{array}$ \\
\hline $\begin{array}{l}\text { Smart Growth } \\
\text { INDEX }\end{array}$ & $\begin{array}{l}\text { Avalia alternativas de transporte e uso } \\
\text { do solo e seu impacto na demanda de } \\
\text { viagem, consumo de solo, habitação, } \\
\text { densidade de emprego e emissão de } \\
\text { poluentes. }\end{array}$ & $\begin{array}{l}\text { O modelo é bem completo em relação às análises } \\
\text { integradas de uso do solo e transportes, incluindo um } \\
\text { módulo próprio de projeção de demanda de transportes, } \\
\text { ou com possibilidade de ser conectado a outros pacotes } \\
\text { específicos de análise de transportes. A alocação } \\
\text { espacial do crescimento previsto não inclui efeitos do } \\
\text { preço do solo e o método de alocação não pode ser } \\
\text { modificado por usuários. Requer uma base de } \\
\text { informações geográficas bastante detalhada. }\end{array}$ \\
\hline Smart Places & $\begin{array}{l}\text { Simula e avalia alternativas de } \\
\text { desenvolvimento do uso do solo e } \\
\text { transportes usando indicadores de } \\
\text { performance ambiental. }\end{array}$ & $\begin{array}{l}\text { Possui ampla integração com sistemas de informação } \\
\text { geográfica e permite avaliar diversos usos e os } \\
\text { impactos recíprocos em diversas áreas, como } \\
\text { transportes, políticas urbanas, situação fiscal e meio } \\
\text { ambiente. Porém, sua flexibilidade implica em menor } \\
\text { sofisticação dos modelos de análise, requerendo que o } \\
\text { usuário forneça além dos dados de entrada, os modelos }\end{array}$ \\
\hline
\end{tabular}




\begin{tabular}{|c|c|c|}
\hline Modelo & Descrição & Características - Vantagens e limitações \\
\hline & & $\begin{array}{l}\text { de avaliação. Além disso, é uma extensão do software } \\
\text { ESRI ArcView GIS e requer o mesmo para operar. }\end{array}$ \\
\hline TRANUS & $\begin{array}{l}\text { Desenvolvido pela Modelística, analisa } \\
\text { os efeitos de uso do solo e políticas de } \\
\text { transporte em variações de localização } \\
\text { de atividades e mercado imobiliário. } \\
\text { Representa o mercado de solo com } \\
\text { preços endógenos em cada período. } \\
\text { (MODELÍSTICA, 2009) }\end{array}$ & $\begin{array}{l}\text { Permite uma vasta quantidade de análises relacionadas } \\
\text { a uso do solo e transportes, permitindo comparar } \\
\text { cenários. O modelo baseia-se em análises econômicas e } \\
\text { relações de consumo entre os setores de atividades } \\
\text { definidos pelo usuário. Requer uma base de dados } \\
\text { detalhada e desagregada. Contempla análises de } \\
\text { transportes, mas não tão sofisticadas quanto um } \\
\text { software específico dessa área, requerendo alguma } \\
\text { análise complementar. A calibração do cenário base é } \\
\text { demorada e difícil de ser alcançada. Em contrapartida, } \\
\text { o desenvolvedor fornece apoio em grupos de discussão } \\
\text { e por e-mail. }\end{array}$ \\
\hline UGrow & $\begin{array}{l}\text { Projeta mudanças de longo prazo em } \\
\text { comunidades em resposta a mudanças } \\
\text { nas políticas fiscais e de transportes. }\end{array}$ & $\begin{array}{l}\text { Embora permita simular diferentes tipos de uso do } \\
\text { solo, as influências da oferta de transportes e das } \\
\text { políticas públicas no uso do solo não são medidas. Não } \\
\text { se trata de uma ferramenta de projeção, atuando mais } \\
\text { no sentido de facilitar o entendimento das relações } \\
\text { entre variáveis. Requer apoio dos desenvolvedores para } \\
\text { implementação. }\end{array}$ \\
\hline UPLAN & $\begin{array}{l}\text { Cria padrões de desenvolvimento } \\
\text { alternativos em resposta a mudanças } \\
\text { nos cenários políticos e de } \\
\text { desenvolvimento. }\end{array}$ & $\begin{array}{l}\text { Permite simular uma variedade de usos, e diferentes } \\
\text { impactos, mas a modelagem e a base teórica não são } \\
\text { sofisticadas e insuficientes para analisar relações entre } \\
\text { variáveis de decisão de planejamento. }\end{array}$ \\
\hline URBANSIM & $\begin{array}{l}\text { Modelo microeconômico de escolha } \\
\text { locacional de residência e emprego. } \\
\text { (WADELL, 2000) }\end{array}$ & $\begin{array}{l}\text { Simula o impacto de decisões chave no } \\
\text { desenvolvimento urbano, principalmente efeitos na } \\
\text { mobilidade e escolhas de localização de moradias e } \\
\text { negócios. A calibração requer conhecimento de } \\
\text { estatística e utilização de softwares auxiliares. Possui } \\
\text { alta resolução espacial e é um modelo de fonte aberta, } \\
\text { podendo ser modificado para se adequar as } \\
\text { necessidades do projeto, mas requer grande quantidade } \\
\text { de dados. }\end{array}$ \\
\hline What if? & $\begin{array}{l}\text { Projeta demanda futura de uso do solo } \\
\text { alocando consumo conforme } \\
\text { adequação da localização. }\end{array}$ & $\begin{array}{l}\text { O software possui uma boa integração com sistemas de } \\
\text { informação geográfica, embora não requeira a } \\
\text { utilização de nenhum outro software complementar. É } \\
\text { flexível em relação aos dados de entrada. O software } \\
\text { permite ao usuário visualizar diferentes cenários, mas } \\
\text { não fornece ferramentas de modelagem para avaliar } \\
\text { fatores inter-relacionados ao desenvolvimento urbano. }\end{array}$ \\
\hline
\end{tabular}

Fonte: elaboração própria a partir de US EPA (2000)

\section{MATERIAIS E MÉTODOS}

A metodologia empregada na pesquisa foi qualitativa. Como alerta Campos (2004), a escolha de método e técnicas para a análise de dados, deve obrigatoriamente proporcionar um olhar multifacetado sobre a totalidade dos dados recolhidos (corpus). Partindo de uma revisão bibliográfica sobre os métodos a serem comparados, sintetizada na Tabela 1, optou-se por adotar um método menos subjetivo para análise comparativa. Um método amplamente utilizado na análise de dados qualitativos é o de 
análise de conteúdo, compreendida como um conjunto de técnicas de pesquisa cujo objetivo é a busca do sentido ou dos sentidos de um documento.

Para a análise empregou-se o software Iramuteq, desenvolvido por Pierre Ratinaud (Lahlou, 2012; Ratinaud \& Marchand, 2012) e licenciado por GNU GPL (v2), que oferece a possibilidade de diferentes formas de análise de dados textuais, tais como (CAMARGO e JUSTO, 2013):

- Análises lexicográficas clássicas: Identifica e reformata as unidades de texto, identifica a quantidade de palavras, frequência média e hapax (palavras com frequência um), pesquisa o vocabulário e reduz as palavras com base em suas raízes (formas reduzidas), cria do dicionário de formas reduzidas, identifica formas ativas e suplementares.

- Nuvem de palavras: a partir da frequência, as palavras são agrupadas e organizadas graficamente.

- Análise de similitude: Esse tipo de análise baseia-se na teoria dos grafos e possibilita identificar as co-ocorrências entre as palavras. Seu resultado traz indicações da conexidade entre as palavras, auxiliando na identificação da estrutura da representação.

- Método da Classificação Hierárquica Descendente

(CHD):
Esta análise visa obter classes de segmentos de texto que, ao mesmo tempo, apresentam vocabulário semelhante entre si, e vocabulário diferente dos segmentos de texto das outras classes. Os segmentos de texto são classificados em função dos seus respectivos vocabulários, e o conjunto deles é repartido em função da frequência das formas reduzidas. A partir de matrizes cruzando segmentos de textos e palavras, em repetidos testes do tipo quiquadrado ( $\chi^{2}$ ), aplica-se o método de CHD e obtémse uma classificação estável e definitiva (Reinert,1990). Além disto, o programa fornece uma outra forma de apresentação dos resultados, através de uma análise fatorial de correspondência feita a partir da CHD. Com base nas classes escolhidas, o programa calcula e fornece-nos os segmentos de texto mais característicos de cada classe (corpus em cor) permitindo a contextualização do vocabulário típico de cada classe.

Para alcançar o objetivo desejado, a aplicação do método seguiu as etapas apresentadas na Figura 1, descritas em seguida: 
Figura 1: Etapas de aplicação do método.

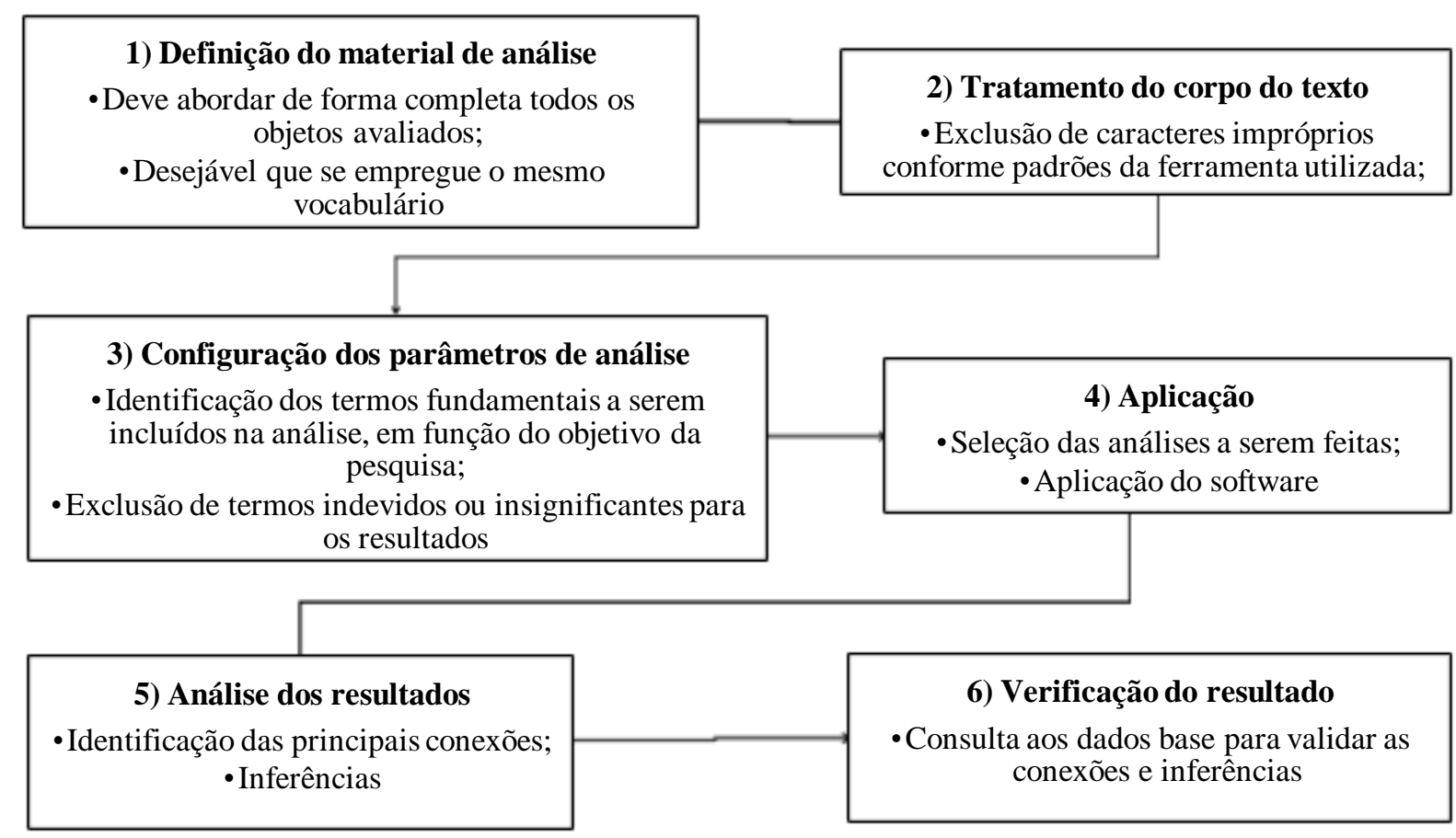

\section{- Etapa 1: Definição do material de análise}

O texto a ser analisado deve ser selecionado de forma a abordar de forma completa todos os objetos avaliados, ou seja, deve trazer o mesmo tipo e detalhamento de informação sobre todas as variáveis investigadas, no caso os modelos. Preocupa-se também com o tipo de vocabulário empregado nas descrições, sendo desejável que se use o mesmo vocabulário ao se referir a cada variável. Essas verificações se mostraram importantes para evitar distorções dos resultados em função da carência de informações ou variações de termos com mesmo significado, diluindo seu peso na avaliação final de frequências.

\section{- Etapa 2: Tratamento do corpo do texto}

Etapa necessária para adequar o texto aos padrões da ferramenta de análise utilizada, contemplando, por exemplo, a exclusão de caracteres impróprios.

\section{- Etapa 3: Configuração dos parâmetros de análise}

Essa etapa ajuda a refinar a análise. Nela são pré-identificados os termos fundamentais a serem incluídos na análise, em função do objetivo da pesquisa. Além disso, os termos indevidos ou insignificantes para os resultados podem ser excluídos das métricas.

\section{- Etapa 4: Aplicação}

Durante a aplicação são selecionadas as análises a serem feitas e verificados os parâmetros requeridos pela ferramenta utilizada.

- Etapa 5: Análise dos resultados

A análise de resultados contempla identificar as principais conexões entre os termos e realizar inferências sobre os significados dessas conexões.

\section{- Etapa 6: Verificação do resultado}

Para verificação dos resultados, as análises feitas foram comparadas às informações completas dos textos base, permitindo validar as conexões e inferências.

O corpo textual foi extraído da publicação EPA (2000), por apresentar o mesmo padrão de 
informações na descrição de cada modelo, evitando assim eventuais distorções da análise em função da variação de vocabulário ou expressões proveniente do texto de diferentes autores. Foram feitas análises sobre a descrição geral das aplicações dos modelos, sobre suas vantagens e suas limitações.

\section{RESULTADOS E DISCUSSÃO}

A seguir serão apresentados os resultados de cada uma das análises de conteúdo.

\section{Descrição geral}

A primeira análise foi feita em relação a descrição geral dos vinte e dois modelos apresentados. A nuvem de palavras resultante da análise de frequência dos termos utilizados na descrição (Figura 2) coloca em destaque as palavras modelo, solo, desenvolvimento, política, urbano, desenvolvimento, crescimento, transporte, entre outros. É possível perceber o foco dos modelos na questão do uso do solo, e as aplicações para avaliar políticas e desenvolvimento. $\mathrm{O}$ transporte não aparece com tanto destaque, mas ainda assim é possível percebê-lo bastante presente na descrição dos modelos. De forma geral as palavras coincidem com as definições mais genéricas de modelos integrados de uso do solo, como apresentado na introdução.

Figura 2. Nuvem de palavras sobre a descrição dos modelos.

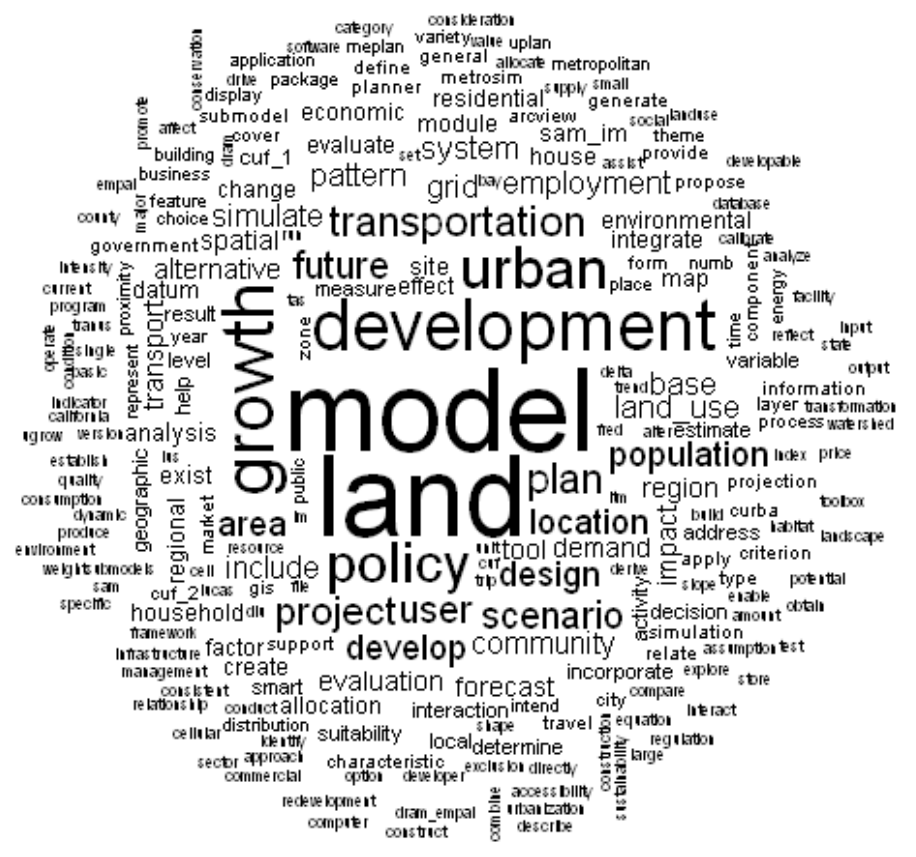

\section{Análise de similitude}

A análise de similitude, Figura 3, permite identificar a conexão de termos. É possível observar dois grandes núcleos de conexão. $\mathrm{O}$ primeiro, relativo à modelagem, ligando-se a diversos descritores do que pode ser simulado pelos modelos, destacando-se política, futuro, transporte, urbano, e a principal conexão com o termo solo. É também identificável 
uma ramificação em relação às possibilidades oferecidas aos usuários dos modelos. O núcleo solos se relaciona principalmente com crescimento, desenvolvimento, plano. $\mathrm{O}$ conjunto de conexões demonstra todas as possibilidades relativas à modelagem do solo, reforçando suas características de apoio à decisão do planejador.

Figura 3. Análise de similitude da descrição geral dos modelos.

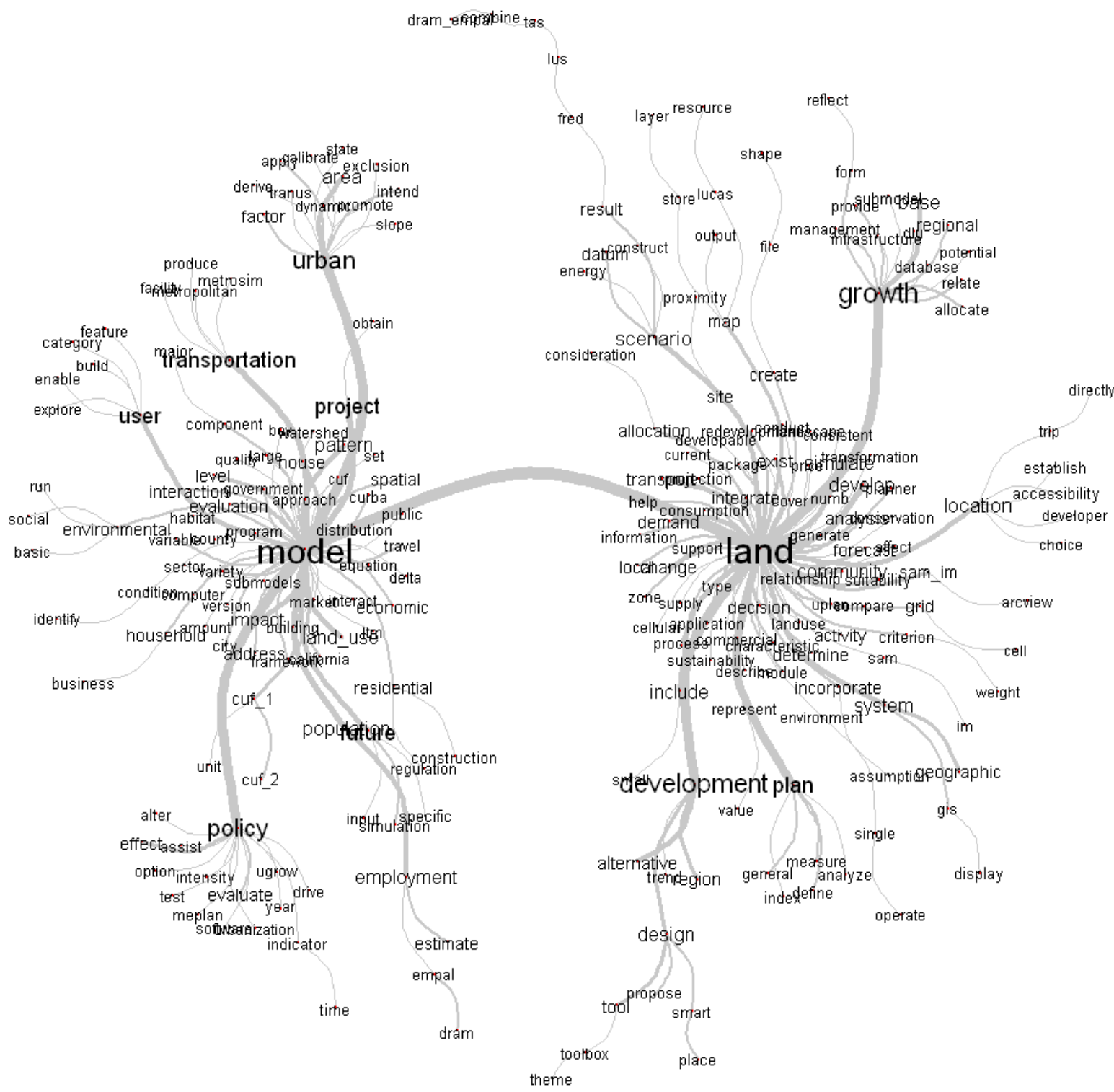

\section{Análise fatorial de correspondência}

A CHD dos modelos distingue sete classes diferenciadas por cores na análise fatorial de correspondência representada na Figura 4. Essa análise define as classes a partir dos grupos de palavras semelhantes, o que permite identificar os modelos que apresentam características descritivas semelhantes entre si. 
Figura 3. Análise fatorial de correspondência dos modelos.

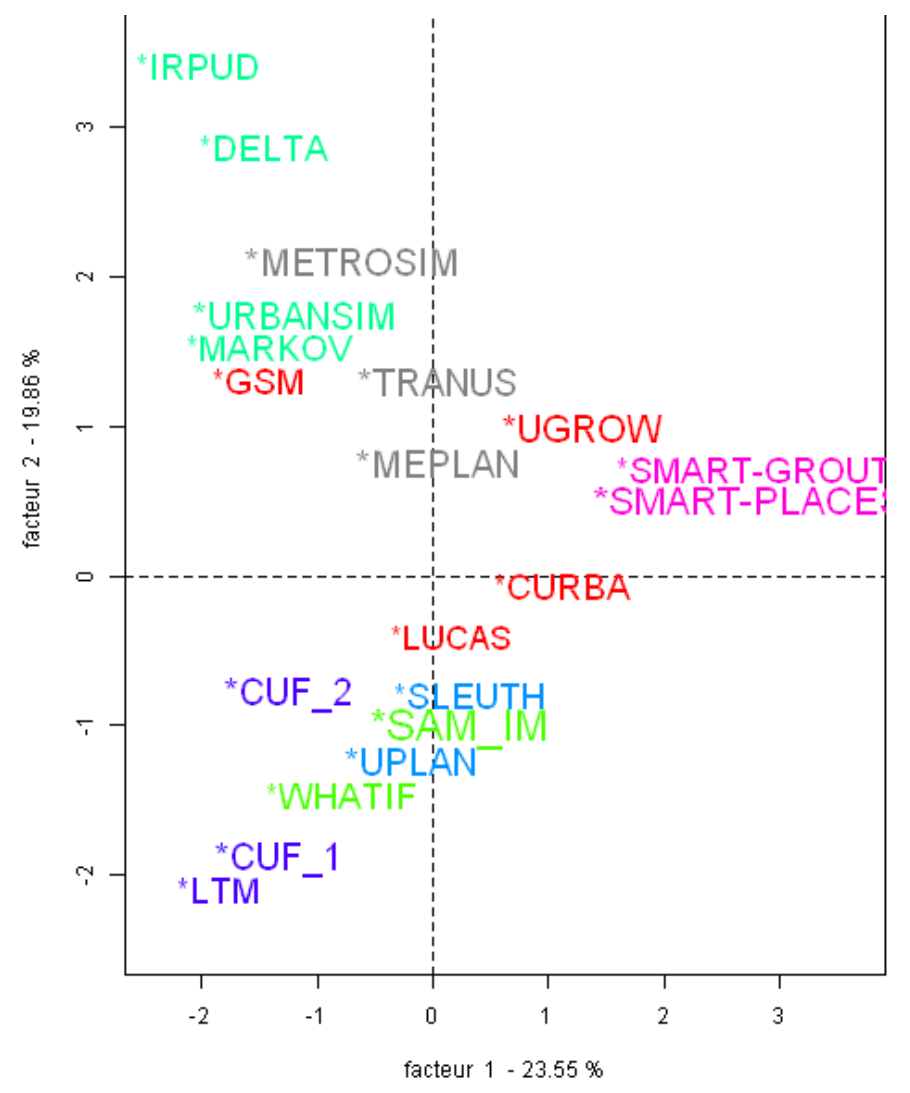

Analisando a representação das classes a partir dos termos utilizados é possível interpretar os agrupamentos feitos (Figura 5). Quanto maior a distância entre as classes, maiores as diferenças entre os termos presentes em cada uma. Os modelos IRPUD, DELTA, URBANSIM e MARKOV, identificados na Figura 4 pela cor verde, apresentam características semelhantes de modelagem dos usos do solo, empregos, moradia, edificações comerciais, e modelagem de demanda e mercado, que são os termos identificados no grupo de mesma cor na Figura 5. Observa-se nesse grupo também o termo regional. Os modelos SMART GROWTH INDEX e SMART PLACES se diferenciam pela utilização de indicadores, meio ambiente e avaliação de cenários. Os modelos CUF-1, CUF-2 e LTM se diferenciam dos demais em relação à questão espacial, característica de submodelos e variáveis.

Os demais modelos se posicionam muito próximos, mas ainda assim diferenciam-se em classes. Os modelos METROSIM, TRANUS e MEPLAN enquadram-se na classe associada aos termos transporte, atividades, metropolitano, suprimentos, projeção, integrado, produção, consumo. Trata-se de modelos em que o problema de transporte se destaca dentre as finalidades, e as relações de produção e consumo são determinantes. Os modelos GSM, CURBA, UGROW e LUCAS se destacam na simulação de cenários futuros. O SLEUTH e o UPLAN apresentam a possibilidade de utilização de dados mais desagregados ao utilizarem sistema de grid e células para o planejamento. O SAM-IN e o 
WHAT IF são modelos que projetam e alocam a demanda de solo de acordo com a afinidade da área.

Figura 4. Análise fatorial de correspondência dos termos utilizados.

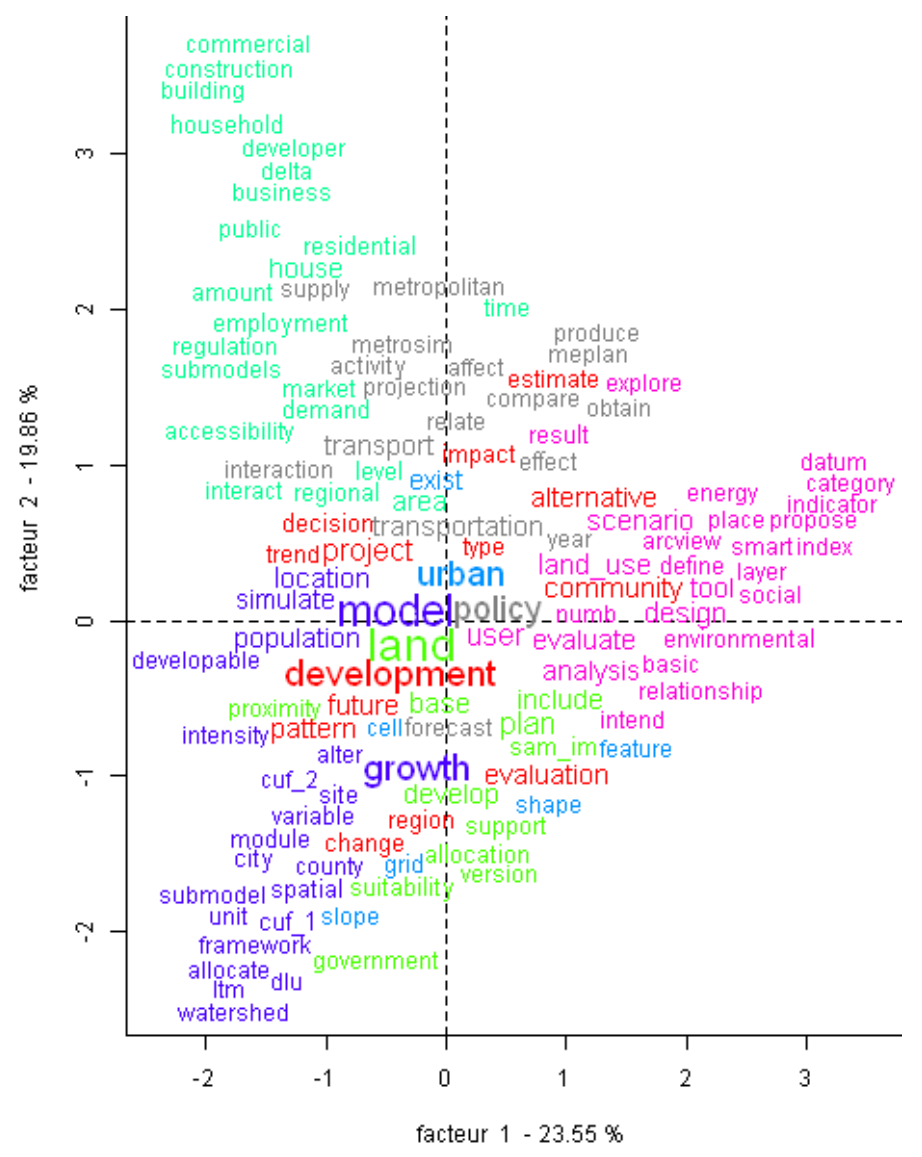

Análise de similitude de vantagens e limitações

Para as vantagens e limitações foi feita apenas a análise de similitude aplicada a todos os modelos de forma agregada. A análise de vantagens (Figura 6) permite identificar sete núcleos: modelo (model), política (policy), alternativa (alternative), cenário (scenario), usuário (user), solo (land), e sistema de informação geográfica (gis). O primeiro reúne características operacionais do modelo e possibilidades de aplicação, tais como simulação e aplicações espaciais.
Uma dessas características, aplicações políticas, desenvolve uma ramificação relacionada à avaliação de alternativas e simulações de cenários. Destacam-se também características de interesse do usuário, como facilidade de implementação em termos de processo e requisitos. Nesse ponto conecta-se a utilização de plataformas de sistemas de informação geográfica (GIS), que permitem associar um banco de dados a mapas digitalizados, como um ponto positivo para manuseio de dados, possibilidade de análises integradas e demais ferramentas complementares. 
Figura 5. Análise de similitude das vantagens.

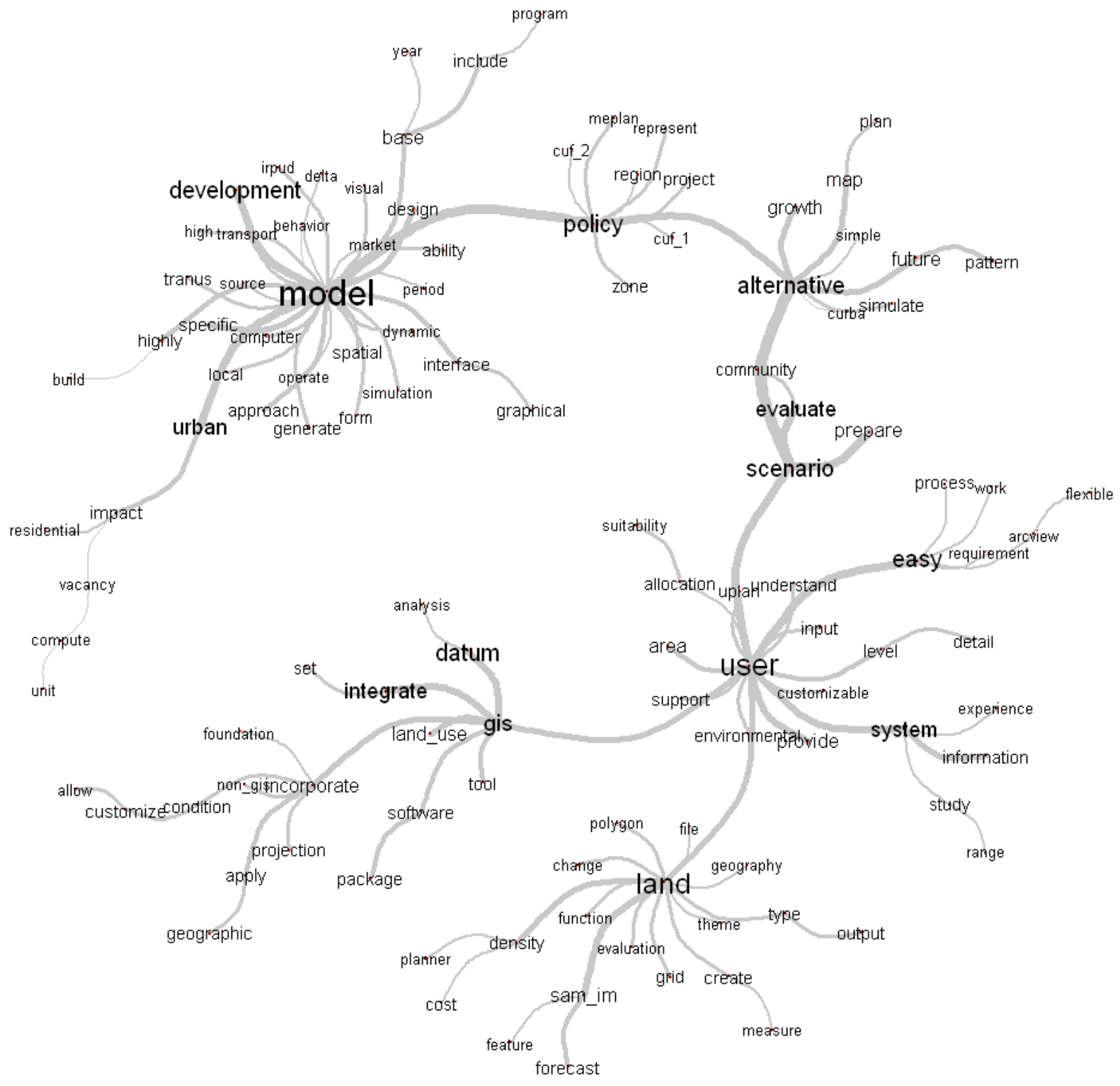

Comparando-se esses resultados com a análise de similitude das limitações (Figura 7) observa-se a ocorrência de muitos termos em comum nas duas análises, porém as conexões são diferentes. Ao termo modelo estão conectadas expressões relacionadas a requisitos e sistemas. $\mathrm{O}$ termo gis se conecta a modelo seguido dos requisitos necessários à modelagem, associados a expressões indicativas de falta ou indisponibilidade. $\mathrm{O}$ termo dados se conecta diretamente a modelo, associado à limitação, cálculo, resolução e tempo, indicando algumas das dificuldades de se trabalhar com os dados no processo de modelagem. $\mathrm{O}$ termo desenvolvimento associa-se a impactos, infraestrutura, padrões e alocação indicando possíveis pontos de dificuldade na modelagem 
Figura 6. Análise de similitude das limitações.

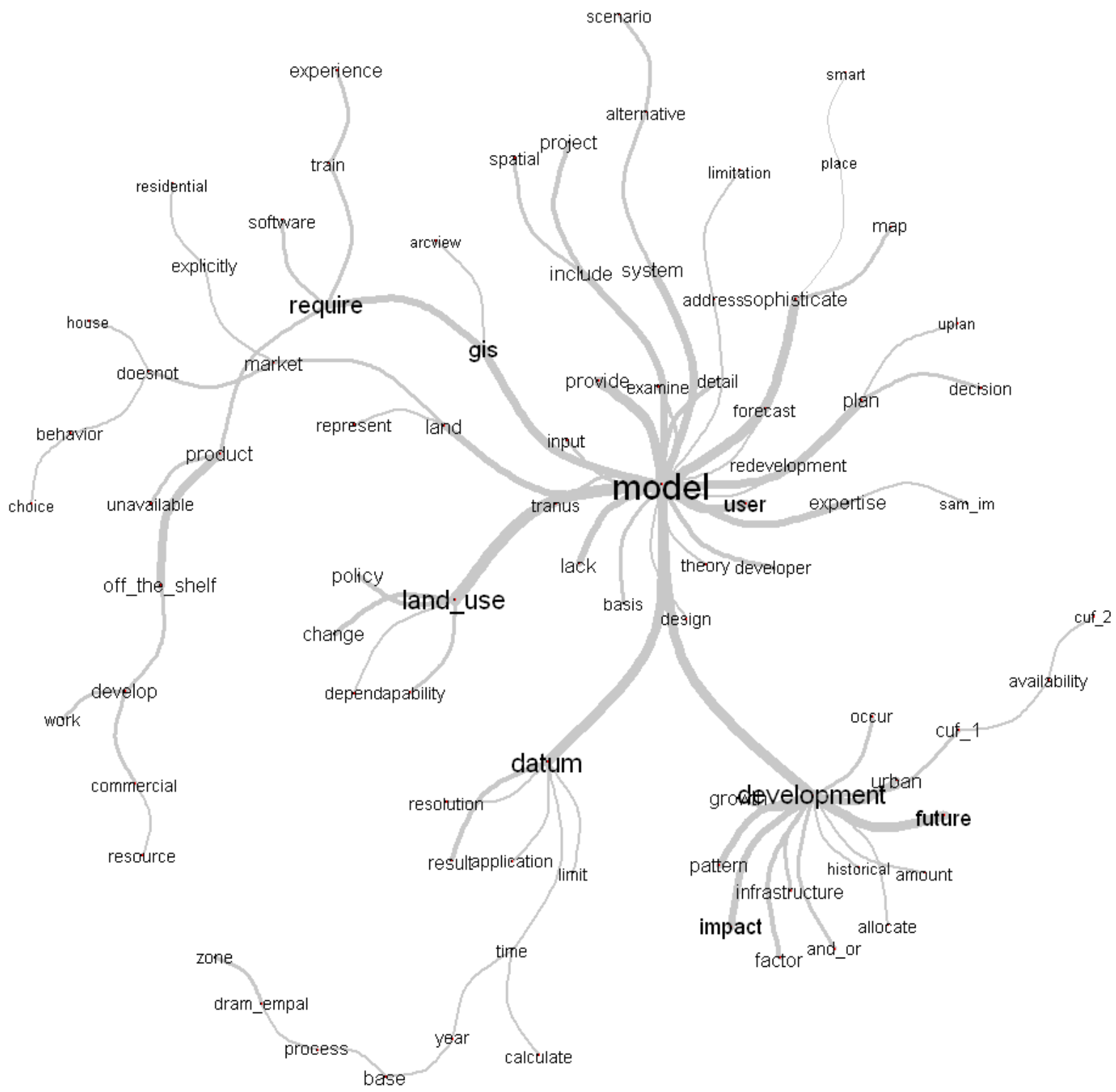

\section{CONCLUSÃO}

Foram empregados métodos de análise de conteúdo para comparar e avaliar vantagens e limitações de vinte e dois modelos integrados de uso do solo e transportes. Por meio dos métodos utilizados foi possível identificar palavras-chave e as conexões entre elas, além de classificar os métodos em grupos conforme a semelhança de suas descrições.
Os resultados obtidos permitem caracterizar de forma geral os modelos analisados e elencar os pontos principais para estudos mais aprofundados, reduzindo significativamente o volume de informações a serem estudadas. Embora inferências mais detalhadas requeiram alguma consulta ao material base, observa-se com o estudo feito que os métodos de análise de conteúdo podem se tornar importantes ferramentas de auxílio ao planejador, no momento de escolha do modelo a ser utilizado, ao 
permitir uma rápida avaliação de um grande volume de informações e direcionar análises mais apuradas para os modelos que mostram mais semelhanças com os objetivos a serem atendidos.

Em relação à interpretação dos resultados, chama atenção a limitação imposta pela disponibilidade e tratamento dos dados necessários à construção de um modelo integrado de uso do solo e transportes. Como apresentado na Tabela 1, os modelos que conseguem flexibilizar os dados de entrada, acabam por simplificar os modelos de cálculo e resultar em análises com menor poder de explicação das interrelações entre variáveis de decisão. Além disso, a frequência com que a palavra urbano aparece nos resultados mostra o perfil e a escala almejada por esses modelos. No entanto, essa aplicação em escala urbana parece agravar as limitações impostas pelas requisições de dados, quanto mais difíceis de obter quanto menor a escala de agregação. Essa constatação sustenta a necessidade de pesquisas futuras acerca da aplicabilidade de modelos integrados para fins regionais.

Todos os autores declararam não haver qualquer potencial conflito de interesses referente a este artigo.

\section{REFERÊNCIAS}

BANDEIRA, J. F. M. Modelo de uso do solo de Aveiro: impacto na mobilidade e qualidade do ar. Dissertação de Mestrado. Departamento de Ambiente e Ordenamento. Universidade de Aveiro. 2009.

CAMARGO, B. V. E JUSTO, A. M. (2013) Tutorial para uso do software de análise textual IRAMUTEQ. Laboratório de Psicologia Social da Comunicação e Cognição - LACCOS. Universidade Federal de Santa Catarina, Brasil.

CAMPOS, C. J. G. (2004) Método de análise de conteúdo: ferramenta para a análise de dados qualitativos no campo da saúde. Revista Brasileira Enfermagem. v. 57, n. 5, p. 611-4.
FEIL, M., M. BALMER AND K. W. AXHAUSEN (2009) New approaches to generating comprehensive all-day activity-travel schedules, Research Report, 575, IVT, ETH Zurich, Zurich.

KRAMER, J. (1996). Integration of A GIS with a Local Scale Self-Modifying Urban Growth Model in Southeastern Orange County, New York. M.A. Thesis. Hunter College-CUNY

LAHLOU, S. (2012). Text Mining Methods: An answer to Chartier and Meunier. Papers on Social Representations, 20 (38), 1-7.

MODELISTICA. A guide to the application of the TRANUS modeling system to the city of Swindon, UK. Modelítica, 2009.

PARSONS BRINCKERHOFF QUADE \& DOUGLAS, INC.; Urban Analytics, Inc. Development and Calibration of the Statewide Land Use-Transport Model. Transportation and Land Use Model Integration Program Phase II, Task 2.3. New Mexico, USA, 1999.

PEREIRA, L. S. F. AND OLIVEIRA, L. K. (2014) Metodologia para estimativa de fluxos de carga a partir de dados secundários: uma aplicação em Belo Horizonte. Journal of Transport Literature, vol. 8, n. 4 , p. 279-315.

RATINAUD, P., E MARCHAND, P. (2012). Application de la méthode ALCESTE à de "gros" corpus et stabilité des "mondes lexicaux": analyse du "CableGate" avec IraMuTeQ. Em: Actes des 11eme Journées internationales d'Analyse statistique des Données Textuelles (835-844). Presented at the 11eme Journées internationales d'Analyse statistique des Données Textuelles. JADT 2012, Liège.

REINERT, M. (1990). ALCESTE, une méthodologie d'analyse des données textuelles et une application: Aurélia de G. de Nerval. Bulletin de méthodologie sociologique, (28) 24-54.

VICHIENSAN, V.; SATO, K.; MIYAMOTO, K.; KITAZUME, K. Introduction of land use model to improve travel demand forecasting in a metropolitan area: A case of Tranus application to Sapporo. Journal of the Eastern Asia Society for Transportation Studies, V. 5. 2003.

U.S. EPA, (2000). Projecting Land-Use Change: A Summary of Models for Assessing the Effects of Community Growth and Change on Land-Use Patterns. EPA/600/R-00/098. U.S. Environmental 
Protection Agency, Office of Research and Development, Cincinnati, OH. 260 p.

WADDELL, P. (2000). A behavioral simulation model for metropolitan policy analysis and planning: residential location and housing market components of UrbanSim. Environment and Planning B: Planning and Design 2000, volume 27(2): 247 - 263. 